\title{
Von der Herstellung struktureller Ungleichheiten und der Erschaffung neuer Handlungsräume
}

\section{Eine qualitative Pilotstudie zur Situation anerkannter Flüchtlinge in peripheren Räumen}

\author{
Elisabeth Scheibelhofer • Sabrina Luimpöck
}

(C) Der/die Autor(en) 2016. Dieser Artikel ist eine Open-Access-Publikation.

Zusammenfassung Der Beitrag beschäftigt sich mit der Frage, in welchen Lebenszusammenhängen Flüchtlinge in Österreich leben, die sich nach einem positiven Ende ihres Asylverfahrens in einem ländlichen Umfeld niedergelassen haben. Die wenigen hierzu existierenden Studien sowie erste eigene Erhebungen (im Rahmen einer im Frühjahr 2016 noch laufenden Antragstellung) deuten darauf hin, dass diese komplexen und vielfältigen Lebensbedingungen in einem Spannungsfeld zu verstehen sind, das von sozialer Ungleichheit geprägt ist und in dem Flüchtlinge dennoch - trotz großteils widriger Umstände - ihre eigenen Handlungsspielräume erschaffen bzw. erweitern können.

Schlüsselwörter Flüchtlinge · Peripherer Raum · Handlungsräume · Qualitative Fallstudie · Soziale Ungleichheit

\section{Manufacturing structural inequalities and the creation of action spaces}

A qualitative pilot study on the situation of recognized refugees in peripheral spaces

Abstract The focus of this contribution is on the question in which circumstances of everyday life refugees are living in peripheral regions after receiving asylum in Austria. The few existing studies and first own investigations (in the context of

In diese Antragstellung ist neben den Partnerinnen aus Deutschland und der Schweiz vor allem Andrea Fritsche involviert. Ihre intellektuellen Beiträge werden wo möglich in diesem Text ausgewiesen. Clara Holzinger danken wir ebenfalls für ihre kritische Lektüre des Manuskripts.

\footnotetext{
E. Scheibelhofer

Department Soziales, FH Burgenland, Campus 1, 7000 Eisenstadt, Österreich

E-Mail: elisabeth.scheibelhofer@univie.ac.at

S. Luimpöck $(\bowtie)$

Institut für Soziologie, Universität Wien, Rooseveltplatz 2, 1090 Wien, Österreich

E-Mail: sabrina.luimpoeck@fh-burgenland.at
} 
project preparation) lead to the assumption that complex and heterogeneous circumstances of everyday life are to be seen in relation to social inequalities specifically harming the positioning of migrants. Nevertheless, the two case studies presented also show how individuals forge out spaces of action in spite of these unfavorable structural conditions.

Keywords Refugees · Peripheral spaces - Action spaces - Qualitative case studies · Social inequality

\section{Zur Lage von Flüchtlingen im peripheren Raum nach dem Asylprozess}

Obwohl Flüchtlinge momentan ${ }^{1}$ das dominante politische und mediale Thema sind, gibt es im deutschsprachigen Raum bis auf wenige Ausnahmen kaum Untersuchungen zur Lage jener Personen, die am Ende ihres Asylverfahrens einen positiven Bescheid erhalten haben. ${ }^{2}$ Diese MigrantInnen sind beziehungsweise werden Teil der österreichischen Gesellschaft, und die Ausgestaltung ihrer Lebenszusammenhänge ist daher nachhaltig von soziologischem Interesse.

Zur Anzahl von Flüchtlingen nach Regionen liegen für Österreich folgende Schätzungen vor: Laut UNHCR (2014) lebten im Jahr 2013 insgesamt 55.598 anerkannte Flüchtlinge und Personen mit subsidiärem Schutz in Österreich. ${ }^{3}$ Trotz der Versuche einer Harmonisierung des Asylwesens innerhalb der $\mathrm{EU}^{4}$ bestehen nach wie vor nationale Unterschiede in Bezug auf Mindeststandards für die Unterbringung von Asylsuchenden. Gemeinsam ist jedoch der weitgehende gesellschaftliche Ausschluss während des Asylverfahrens. Trotz oder gerade wegen der EU-weiten Unterschiede erscheint der exklusiv nationale Blick auf die Thematik nicht mehr adäquat. Es wird vielmehr ein Forschungsansatz notwendig, der zugleich eine transnationale sowie regional vergleichende Perspektive ermöglicht. Der Großteil der vorliegenden Untersuchungen konzentriert sich auf jene geographischen Gebiete innerhalb Europas,

\footnotetext{
${ }^{1}$ Dieser Beitrag wurde zwischen Sommer 2015 und Frühjahr 2016 verfasst, was im Kontext der Debatte um Flüchtlinge wohl eine wichtige Zusatzinformation darstellt.

2 Für Österreich werden in diesem Beitrag zu dieser Gruppe sowohl subsidiär Schutzberechtigte als auch Personen, die Asyl erhalten, gezählt. Dies ist insofern zu rechtfertigen, als die Rechte dieser beiden juristisch unterschiedenen Gruppen im Jahr 2011 zu einem Großteil angeglichen wurden (Servent et al. 2014, S. 323; European Parliament and Council of the European Union 2011).

${ }^{3}$ Diese Schätzung ergibt sich aus den Daten zur individuellen Zuerkennung eines Asylstatus von 2001-2011 (Statistik Austria, 2012, zitiert nach UNHCR 2014, S. 13). So wurden im Jahr 201316.675 Anträge auf Asyl entschieden (BMI 2014), von welchen 4133 oder 24,8\% positiv ausfielen (hier sind auch Entscheidungen für subsidiären Schutz mit eingerechnet) (ebd.). Diese Anerkennungsraten werden aller Voraussicht nach aufgrund der kriegerischen Auseinandersetzungen in den Herkunftsländern der derzeit in Österreich ankommenden Flüchtlinge weiterhin steigen.

4 Das Asylwesen wurde auf EU-Ebene in den letzten Jahren durch die Einführung des Common European Asylum System (CEAS) 1999 bzw. 2003 verändert (European Commission 2014). Trotz der damit intendierten Harmonisierung des europäischen Asylwesens kam es jedoch seither nur zu geringen Änderungen der Minimalstandards in der Behandlung von Asylsuchenden innerhalb der EU (Servent et al. 2014; Scheibelhofer 2016; Reichel und Cibea 2014).
} 
in denen sich die meisten Flüchtlinge nach ihrem positiven Bescheid niederlassen und das sind bekanntermaßen die städtischen Ballungsräume. Obwohl diese Fokussierung angesichts der ungleichen Ansiedlung gut begründet ist, ${ }^{5}$ scheint es dennoch angebracht, vergleichend die Situation im ländlich-peripheren Raum in den Blick zu nehmen (eine der wenigen Studien hierzu wurde von Brunner et al. 1994 vorgelegt).

Für die vorliegende Studie wurde das südliche Burgenland als Untersuchungsregion gewählt, da die allgemein vergleichsweise schwache Infrastruktur und die geringe Verbreitung von einschlägigen NGOs die periphere Lage für Flüchtlinge in dieser Region noch akzentuiert - obgleich das Bundesland historisch stark von Migration geprägt ist. Die heutige autochthone kroatische Minderheit etwa geht auf Umsiedlungen aus dem 16. Jahrhundert zurück. Mitte des 19. Jahrhunderts setzte aufgrund der schlechten wirtschaftlichen Lage außerdem eine starke Emigration in die USA ein. Heute ist das Burgenland attraktiv für PendelmigrantInnen aus angrenzenden EU-Staaten. Der Anteil der Bevölkerung, der aus den wichtigsten Herkunftsländern von Flüchtlingen stammt, ist jedoch vergleichsweise gering.

Nicht nur Regionen, in denen sich Flüchtlinge niederlassen, haben ihre Spezifika, auch Flüchtlinge selbst bringen spezifische Erfahrungen mit und sind oftmals vor bzw. während ihrer Flucht mit diversen Schwierigkeiten (darunter auch Gewalterfahrungen) konfrontiert. Die empirische Forschung der letzten Jahre hat gezeigt, welch umfassende Wirkung darüber hinaus der Asylprozess an sich auf MigrantInnen hat (Täubig 2009). Die Lebensbedingungen in Massenunterkünften, die Notwendigkeit des Abwartens (Fritsche 2012), der Ausschluss vom Erwerbsleben und die geringen Möglichkeiten der Teilhabe an der Konsumgesellschaft charakterisieren demnach ihre Lebensumstände. Die Annahme liegt nahe, dass diese mitunter jahrelang andauernden Verhältnisse Auswirkungen auf das Alltagsleben nach einem positiven Bescheid haben. So belegen Studien bereits, dass viele anerkannte Flüchtlinge Schwierigkeiten haben, Arbeitsplätze und Wohnraum zu finden (UNHCR 2013, S. 32; Neuwirth 2007; Ammer 2011; Kraler et al. 2013, S. 103). Insbesondere die Nichtteilnahme am Arbeitsleben führt dabei häufig zu Außenseiterpositionen (Burtscher 2012). Analog dazu ist Arbeit als Funktionssystem für Inklusion an oberster Stelle in Diagnostikinstrumenten der Sozialen Arbeit angeführt (Pantuček 2012). Dies verdeutlicht die Exklusion, in die Asylsuchende aufgrund ihres legalen Status gedrängt werden, und die paradoxe Situation in Bezug auf das Inklusionsziel nach der Asylgewährung, in der sich sowohl die Flüchtlinge selbst als auch betreuende Institutionen wiederfinden (Luimpöck 2015).

Doch wie lassen sich diese Effekte aus der Perspektive der Sozialwissenschaft begreifen? Es scheint überzeugend, davon auszugehen, dass MigrantInnen, die als Asylsuchende oder Flüchtlinge bezeichnet werden, von der sog. autochthonen Bevölkerung separiert und zu „Anderen“ gemacht werden. Diese Differenzierung funktioniert auf Grundlage einer ethnischen bzw. nationalen oder kulturell-religiösen Zuschreibung des Andersseins (Jenkins 1997). Um diese Annahmen zu dekonstruieren, hat sich eine dekoloniale Perspektive in der Flüchtlingsforschung als hilfreich erwiesen (Ha 2014), die das Asylsystem als ein System der Reproduktion sozialer

\footnotetext{
${ }^{5}$ In Gemeinden bis 2500 EinwohnerInnen sind nur 6,7\% der Bevölkerung im Ausland geboren, während es in Städten mit über 50.000 EinwohnerInnen $22 \%$ sind (Bundesministerium für Inneres 2014).
} 
Ungleichheit versteht, das Unterschiede erzeugt und verstärkt, die schließlich zu einer ethnisierenden und rassistischen Gesellschaftsordnung führen. Die Stärke eines dekolonialen theoretischen Zugangs (Ha 2014; Sonn und Quayle 2013; Chakrabarty 2000; Conway 2011; Nayar 2010) liegt darin, folgendes Paradoxon zu erklären: Eine Vielzahl sog. ,reicher“ Länder etablieren global gesehen ein Asylwesen, das Personengruppen erzeugt, die zwar langfristig im jeweiligen Aufnahmeland bleiben, denen aber - auch nach positivem Asylbescheid - die Inklusion letztendlich verwehrt bleibt.

\section{Zwischen Differenz und Diskriminierung: Empirische Andeutungen aus der Pilotphase des Forschungsprojektes}

Auf Basis dieser Überlegungen stellt sich die Frage nach den Lebenswelten von Flüchtlingen im ländlichen Raum, die kürzlich einen positiven Asylbescheid erhalten haben. Um die Strategien des Umgangs mit diesen spezifischen Bedingungen zu analysieren, muss die Forschung Flüchtlinge als AkteurInnen begreifen. Aus diesem Grund wurde ein interpretativer Forschungszugang gewählt, der auf einer konstruktivistischen Form der Grounded Theory beruht (Charmaz 2006). Bislang liegen erste Interpretationen vor, die aus der Pilotstudie eines in Antrag befindlichen Projektes stammen (vgl. Fußnote 1). Da die sozialwissenschaftliche Forschung in diesem Bereich noch wenig empirische Ergebnisse vorweisen kann, haben wir uns entschlossen, bereits zum jetzigen Zeitpunkt Einblick in unseren Forschungsprozess zu geben.

Im Folgenden sollen die Fallbeispiele zweier Familien nachgezeichnet werden, die 2012 in deren Zuhause interviewt wurden. Sie wurden dem Forschungsvorgehen der Grounded Theory entsprechend im Zuge eines theoretischen Samplings (Hermanns 1992, S.116) ausgewählt. Die Auswahl gründete auf dem Forschungsinteresse, welches die alltäglichen Lebenswelten von Flüchtlingen im ländlichen, peripheren Raum fokussiert. Aufgrund der Interpretationen der ersten Interviews wurde das Forschungsinteresse auch auf jene Strategien erweitert, die Flüchtlinge im ländlichen Raum einsetzen, um ihre Handlungsräume zu erweitern. Bei der Auswahl der Interviewten wurde - im Sinne der Kritik des ,methodologischen Nationalismus" - nicht eine spezifische Herkunftsregion fokussiert, sondern ein - bezogen auf den Herkunftskontext - vergleichender Ansatz gewählt. Außerdem wurde entschieden, ausschließlich Familien einzubeziehen, um in diesem Kontext Geschlechter- und Generationenverhältnisse zu analysieren. Das theoretische Sampling sah weiters vor, in beiden Fällen mehrere Familienmitglieder zu interviewen, die möglichst unterschiedliche Sozialisationen - bezogen auf Herkunfts- und Ankunftsland - durchlaufen haben sollten. 
Die Kontaktaufnahme mit den beiden interviewten Familien ${ }^{6}$ erfolgte über die Mitarbeiterin einer NGO, die in dieser Region tätig ist. Ihr wurde das Forschungsinteresse geschildert, woraufhin sie eine der beiden Familien gleich zu Gesprächsbeginn empfahl. Die zweite Familie wählte sie erst nach einem längeren Austausch über die Besonderheiten der ersten Familie (Wohnort, Berufstätigkeit, Herkunftsland) für eine Empfehlung an uns aus. Obwohl der Feldeinstieg über die NGOMitarbeiterin für uns sehr hilfreich war, so waren damit auch Selektionskriterien jenseits des theoretischen Samplings verbunden, die es an dieser Stelle offenzulegen gilt, da ihr Einfluss auf die Ergebnisse nicht zu unterschätzen ist. Während des Gesprächs überlegte sie laut, dass sie keine Familien vermitteln wolle, die von der Mindestsicherung lebten und sich mehr oder weniger ,aufgegeben“ hätten. Sie erklärte auch ihre Überlegungen: Es gebe zwar Flüchtlinge, auf die diese Beschreibung zutreffe, sie gäben jedoch nicht das gesamte Spektrum anerkannter Flüchtlinge und subsidiär Schutzberechtigter wieder. Somit gehen wir davon aus, dass uns in dieser Pilotphase sozusagen „Erfolgsgeschichten“ zugespielt wurden. Dies wird in künftigen Forschungszyklen jedenfalls zu berücksichtigen sein. Darüber hinaus ist die Einschätzung der Mitarbeiterin in ihrem Arbeitskontext zu sehen und stellt wertvolles Datenmaterial dar. ${ }^{7}$

\section{Empirisches Fallbeispiel I: Familie Dadrian}

Das Haus von Familie Dadrian ${ }^{8}$ befindet sich am Rand eines Dorfes, das etwa 1000 EinwohnerInnen zählt. Es liegt in einer Gasse mit Einfamilienhäusern, die vermutlich in den späten 1970er und 1980er-Jahren errichtet wurden. Die Straße ist ruhig, Parkplätze gibt es genug, da die Autos direkt vor den Einfamilienhäusern und wohl auch in dazugehörigen Garagen parken. Als ich das Auto vor dem Haus abstelle, merke ich, wie sich in einem Haus auf der gegenüberliegenden Straßenseite ein Fenstervorhang bewegt. Eine Frau um die fünfzig Jahre in Jeans öffnet die Tür. Unser Treffen findet erst einige Wochen nach dem ersten Telefonat statt, weil Frau Dadrian gerade dabei war, die Küche zu renovieren. Nun schildert sie

\footnotetext{
${ }^{6}$ Die methodologische und methodische Reflexion kann an dieser Stelle auf wichtige Themen, wie etwa die Mehrsprachigkeit im Interview (Fritsche 2012), nur verweisen. Auch die Frage der Möglichkeit, eine Kommunikationssituation herzustellen, die auf Vertrauen und Offenheit unter Bedingungen der sozialen Ungleichheit basiert, ist für die qualitative Forschung hier in Zukunft noch weiter zu thematisieren (Kirpitchenko und Voloder 2014).

${ }^{7}$ Interessant ist zum jetzigen Zeitpunkt etwa die Gegenüberstellung mit den - wenn auch spärlich - zugänglichen Daten zu BezieherInnen der sog. bedarfsorientierten Mindestsicherung (BMS). Hierzu lassen die Sozialberichte des Burgenlandes folgende Rückschlüsse zu: Mit Stichtag Ende April 2013 haben insgesamt 2377 Personen im Burgenland BMS bezogen, was einem Anteil von 0,83\% an der burgenländischen Gesamtbevölkerung entsprach. Weiters enthält der Bericht Angaben zur Staatsbürgerschaft: $76 \%$ der BMS-BezieherInnen waren demnach ÖsterreicherInnen, $9 \%$ kamen aus EU-Staaten und $15 \%$ aus sog. „sonstigen Staaten“. Da davon auszugehen ist, dass die hier interessierenden Flüchtlinge (noch) nicht die österreichische Staatsbürgerschaft erlangt haben, handelt es sich um einen Teil jener Personen, die den $15 \%$ der 2377 Personen (oder 357 Personen) angehören, die keine EU-Staatsbürgerschaft haben (Amt der Burgenländischen Landesregierung 2013).

${ }^{8}$ Namen wurden anonymisiert, und auch Details, die die Personen zuordenbar machen würden, wurden im Folgenden entsprechend verändert, sofern sie die Nachvollziehbarkeit der Analyse nicht gefährden.
} 
stolz, welch ein immenser Arbeitsaufwand damit verbunden gewesen sei, den sie mit ihrem Mann und ihren Kindern erfolgreich bewältigt habe. Sie erzählt, dass sie Renovierungsarbeiten generell selbst machten - auch Dinge, die sie nie gelernt oder zuvor ausprobiert hätten. Auch eine neue Kücheneinrichtung wurde eingebaut, es ist eine bekannte Markenküche, von der sie während des Interviews noch ausführlich erzählt. Sie habe sie als Schnäppchen bei einem Händler gekauft. Bevor wir das Interview beginnen, führt mich Frau Dadrian durch das gesamte Haus und den Garten. Am Dachboden erzählt Frau Dadrian, dass es das gemeinsame Hobby mit ihrem Mann sei, auf Flohmärkte und Sperrmüllplätze zu fahren. Oft böten ihnen Leute ausrangierte Dinge an, die Frau Dadrian gerne annehme. Die meisten Gegenstände seien noch gut in Schuss und von besserer Qualität als Waren von Diskontern - wie etwa die vier Staubsauger, die am Dachboden nebeneinander liegen. Auch Kleidung nehme Frau Dadrian und gebe sie an eine Freundin im Herkunftsland weiter. Die Analyse des Beobachtungsprotokolls sowie einiger Interviewpassagen legt nahe, dass Frau Dadrian großen Wert darauf legt, nicht verschwenderisch zu leben. Sparsamkeit mit Ressourcen aller Art ist ein wichtiger Wert für sie. Diese Einstellung hat es ihr und ihrer Familie auch ermöglicht, sich trotz widriger Umstände ein gutes Leben aufzubauen. Diesen sparsamen Umgang verbindet Frau Dadrian - nach unseren Analysen - nicht mit Entbehrung, sondern als einträgliche und zielführende Grundhaltung. So schildert sie etwa, wie sehr ihr das Handeln auf Online-Plattformen Freude mache. Stolz zeigt sie auf ein Armband, das ihre Tochter trägt. Sie hat es vor einiger Zeit günstig am Flohmarkt erstanden und später entdeckt, dass es für ein Vielfaches im Internet zum Verkauf angeboten wurde.

Bevor das Interview in der Wohnküche beginnt, stellt mir Frau Dadrian ihren Mann vor. Im Gegensatz zu seiner Frau ist Herr Dadrian in dieser Situation nicht sehr gesprächig. Bereits beim Rundgang durch das Haus fiel auf, wie häufig Frau Dadrian von gemeinsamen Tätigkeiten mit ihrem Mann sprach: Das Abklappern von Flohmärkten und Sperrmüllplätzen am Wochenende scheint zu ihren gemeinsamen Hobbys zu zählen, das sich auch finanziell lohnt. Da Herr Dadrian aufgrund einer Gehbehinderung hinkt, arbeitet er „nur“ zweimal die Woche als Taxifahrer, wie Frau Dadrian einschränkt. Ansonsten helfe er im Haushalt, wenn Frau Dadrian arbeite. Sie ist in einem Hotel mit angeschlossenem Restaurant als Reinigungskraft und Küchenhilfe beschäftigt. Sie erwähnt zu einem späteren Zeitpunkt, dass sie zu Hause vorkocht, bevor sie in die Arbeit geht, und dass ihr Mann für sich selbst und die beiden jugendlichen Kinder (17 bzw. 19 Jahre alt) das Essen aufwärmt. Bei der Interpretation fällt auf, mit welch ausführlichem Lob für ihren Mann sie diese Tätigkeit verbindet, ohne weitere Beispiele für Unterstützung zu nennen. Wären die Rollen anders verteilt, würde ein derartiges Hervorheben für die Mithilfe der Frau im Haushalt in dieser sozialen Situation unter Umständen anders ausfallen. Hier wird deutlich, dass Frau Dadrian von einem konservativen Geschlechterverhältnis auszugehen scheint, demzufolge die Frau prinzipiell für die Reproduktionsarbeit zuständig ist. Die Leistung des Mannes wird von ihr sehr hoch geschätzt, obwohl sie im Vergleich zur Reproduktionsarbeit, die sie selbst in Haus und Garten zu leisten scheint, minimal sein dürfte. Dies wird an der hohen Bedeutung deutlich, die sie dem Aufwärmen von Speisen beimisst, das ihr Mann für sich selbst und seine zwei jugendlichen Kinder in ihrer Abwesenheit durchführt. 
Die Tochter setzt sich während des Gesprächs zwei Mal zu uns, sie wirkt aufgeschlossen und selbstbewusst. Zu einem späteren Zeitpunkt meines Besuchs kommt sie in einem Brokatdirndl in die Wohnküche, da sie zu einem Konzert ihrer Blasmusikkapelle aufbricht. Die Tochter spricht mit der Mutter burgenländischen Dialekt und Farsi. Der Sohn grüßt von weitem im Gang. Frau Dadrian erzählt, dass er nur schwer Freunde finde und sie ihm immer beigebracht habe, sich von den Schulkameraden nicht ärgern zu lassen.

Die Familie ist zum Zeitpunkt des Interviews seit 13 Jahren in Österreich. Sie war aus dem Iran geflüchtet, da es für sie als Christen die Möglichkeit gab, sich für ein US-Visum zu bewerben. Diese Möglichkeit stand nach den Terroranschlägen 2001 jedoch nicht mehr offen, als die Familie bereits in Österreich war, wo sie um Asyl ansuchten und dieses nach fünf Jahren erhielten. Während dieser Wartezeit lebte die Familie 20 Monate in Wien und schließlich in einem zur Unterkunft für AsylwerberInnen umfunktionierten Gasthof im Südburgenland. Im Rückblick erzählt Frau Dadrian von der Ankunft in Österreich. Nach einer Zeit in der Erstaufnahmestelle Traiskirchen seien sie im Haus einer Hilfsorganisation in Wien untergebracht gewesen, wo BesucherInnen von den MitarbeiterInnen immer in ihre Wohnung gebracht worden seien, weil sie bereits damals Wert auf eine ansprechende Einrichtung gelegt hätten. Die dazu nötigen Möbel hätten sie vom Sperrmüll besorgt oder direkt von NachbarInnen des Wiener Nobelbezirks erhalten, in dem diese Unterkunft lag. Nach einer Auseinandersetzung mit einer anderen Familie in dem Heim sei die Familie gegen ihren Willen ins Burgenland verlegt worden. Frau Dadrian schildert, dass sie damals viel geweint habe und verzweifelt gewesen sei, so weit von einer Stadt entfernt leben zu müssen. Doch mit der Zeit habe sie sich daran gewöhnt, und inzwischen wolle sie nicht mehr wegziehen.

Auf meine Nachfrage, woran das liege, antwortet Frau Dadrian, dass sie sich im Ort so frei fühle, hier könne sie alles machen. Als Beispiele nennt sie Gartenarbeiten. Wien hingegen sei immer stressig. Sie erzählt, dass sie sich zwar beobachtet fühle, aber dass sie das nicht störe: „Ich schaue auch gerne hinüber zu ihnen!“, lacht sie. Die Analyse des Interviewmaterials zeigt, dass eine derart humorvolle und selbstbestimmte Umgangsweise typisch ist für Frau Dadrians Umgang mit negativen, auch als rassistisch zu qualifizierenden Erfahrungen im Umgang mit NachbarInnen oder anderen Personen, mit denen sie in Berührung kommt. Dies wird auch deutlich in der ausführlichen Schilderung des Unglaubens und Neids der ansässigen Dorfbevölkerung, als die Familie ihr Haus zunächst bezog und schließlich erwarb. Im Interview wählt sie lachend den Vergleich mit einer Mafia, als sie erzählt, wie es zur Vermietung kam: Jeder sei hier mit jedem verwandt, und ihr Vermieter habe daher gemeint, sie sollten erst in das Haus einziehen, wenn er in die USA ausgereist wäre. Frau Dadrian deutet dieses Vorkommnis als Angst des Vermieters vor seiner ortsansässigen Verwandtschaft - und hebt somit ihren eigenen Zugang zu Anfeindungen, die auch sie erlebt, hervor. Allerdings hat auch Frau Dadrians offensiv positiver Umgang seine Grenzen. Es ist verstörend für sie, wenn sie sich Verdächtigungen ausgesetzt sieht, die Familie könne die Mittel für ihren Lebensstandard nicht rechtschaffen erworben haben. So erzählt sie im Interview von Unterstellungen der NachbarInnen, wonach Hauskauf und Renovierungen mit ihrem Budget nicht zu machen seien. Frau Dadrian deutet dies als Neid und geht sofort dazu über, ihre eigenen Wertigkeiten 
herauszustreichen: Es gehe darum, fleißig und sparsam zu sein sowie Dinge mit viel Eigeninitiative anzugehen.

Frau Dadrian schafft es mit diversen Strategien, Ressourcen aus diesem - mitunter feindlichen - Umfeld zu erschaffen. $\mathrm{Zu}$ diesen Strategien gehören das Einsammeln (und Wertschätzen) von aussortierten Objekten oder der Aufbau vielfältiger, tragfähiger sozialer Beziehungen, die sie aktivieren kann. So wurde der Bankbeamte, bei dem sie seit Jahren Kredite aufnimmt und zurückzahlt, inzwischen zu einem guten Freund. Ihn kann sie auch bei Problemen um Hilfe bitten, die nichts mit Finanzgeschäften zu tun haben. Frau Dadrian kommt immer wieder auch auf andere autochthone Personen zu sprechen, zu denen sie ein sehr gutes Verhältnis hat, wie beispielsweise ihre jetzige Arbeitgeberin. Auch mit dem Besitzer jener Flüchtlingsunterkunft, in der sie vor Jahren gelebt haben, hat Frau Dadrian nach wie vor regelmäßigen Kontakt. Sie erwähnt eine Reihe von Freundinnen und Arbeitskolleginnen, die allesamt autochthone ÖsterreicherInnen sind. Religiöse bzw. ethnische Netzwerke hingegen sind für Frau Dadrian und ihre Familie kaum relevant. Das zeigt sich im Interview daran, dass Frau Dadrian diese selbst nicht thematisiert und auf Nachfrage hin ausführt, dass ihr der Weg nach Wien zur nächstgelegenen religiösen Vereinigung zu weit ist.

Der Blick auf die eigenen Lebensumstände ist weder naiv noch beschönigend. Sie beschreibt auch Hürden, wenn sie auf ihre Biographie in Österreich zurückblickt. Besonders schwierig sei für sie die Situation gewesen, als sie noch keinen Führerschein besessen und Arbeit gesucht habe. Da das AMS die Kosten nicht übernahm, hat Frau Dadrian zwei Jahre lang gespart, um sich die nötigen Fahrstunden und Prüfungsgebühren leisten zu können.

\section{Empirisches Fallbeispiel II: Familie Bisultanov}

Familie Bisultanov lebt in einer Einkaufsstraße im Zentrum einer Stadtgemeinde mit etwa 4500 EinwohnerInnen. Viele der kleineren Geschäftslokale sind geschlossen bzw. zur Vermietung angeschrieben. Die siebenköpfige Familie wohnt in einer ca. $60 \mathrm{~m}^{2}$ großen Wohnung im Dachgeschoß eines Mehrparteienhauses. Das Interview findet im Wohnzimmer statt, wo ein großer Esstisch an der Wand steht, daneben lehnen Klappstühle an der Wand. Frau Bisultanov deutet mir, mich auf die dem Tisch gegenüberliegende Couch zu setzen. Sie serviert Kaffee und Kuchen, ihr Mann kommt mit verschlafenem Blick aus dem Nebenraum, in dem ich einen Fernseher und einen PC sehe. Ein weiteres Zimmer könnte ein kleines Kinder- oder Schlafzimmer sein. Der dreizehnjährige Sohn ist in der Schule, ihn treffe ich erst bei meinem zweiten Besuch. Der zehnjährige Sohn setzt sich manchmal zu uns und hört zu, ebenso die zwanzigjährige Tochter mit ihrer zweijährigen Tochter.

Das Gespräch verläuft hauptsächlich zwischen Herrn Bisultanov und mir, wobei seine Frau immer wieder bestätigend einsteigt und fast durchgehend bei uns sitzt. Hauptthema seiner Schilderungen sind seine vielfältigen und umfangreichen Anstrengungen, einen Job zu finden. Auch die erfolgreich abgeschlossenen Kurse sowie ein AMS-finanzierter Gabelstapelführerschein hätten nichts genützt. Herr Bisultanov erzählt von Erfahrungen während der Jobsuche und überlegt im Interview 
gemeinsam mit seiner Frau, was die Gründe für dieses - von ihm als solches aufgefasste - Scheitern sind. Herr Bisultanov habe potentiellen Arbeitgebern angeboten, einen Monat lang unbezahlt für sie zu arbeiten, um ihnen so seine Zuverlässigkeit und Kompetenz zu beweisen. Doch auch diese Strategie sei erfolglos geblieben. Er nimmt an, dass sein Alter - er ist schätzungsweise Ende Vierzig - die Arbeitssuche derart erschwert. Er erzählt von jüngeren Personen mit ähnlicher Biographie, die rasch eine Arbeit gefunden hätten. Aufgrund seiner ausführlichen Schilderungen überrascht es, dass er nicht rassistische Gründe in Erwägung zieht. Dies könnte eine Strategie sein, sich nicht selbst als Fremder zu stigmatisieren und auszugrenzen.

Auch Frau Bisultanov erzählt, dass sie gerne wieder arbeiten würde. Sie ist inzwischen sogar bereit, in das zwei Stunden entfernte Wien zu pendeln. Vor der Flucht arbeitete sie als gelernte Köchin. Die Familie lebte nahe der Hauptstadt in einem Ort, wo Herr Bisultanov als Automechaniker und Taxifahrer arbeitete. Bevorzugt würde Frau Bisultanov wieder in ihrem erlernten Beruf arbeiten, allerdings spricht sie davon zurückhaltend, wie von einer kaum erfüllbaren Wunschvorstellung. Tatsächlich bewirbt sie sich für Hilfsarbeiten aller Art. Einschränkend meint sie, dass ihr Deutsch nach wie vor wohl zu schlecht sei und ihre Bemühungen daher scheiterten. Die Anforderungen potentieller DienstgeberInnen etwa thematisiert sie nicht, genauso wenig wie mögliche strukturelle Diskriminierungen. Vielmehr sucht sie den Grund bei sich selbst - ähnlich wie ihr Mann. Während sie jedoch ihre Deutschkenntnisse als veränderbar wahrnimmt, gerät er durch die (im Licht der einschlägigen Forschung wohl berechtigte) Annahme, sein Alter sei ein Hinderungsgrund, in eine passive Position. Ihre mündliche Ausdrucksfähigkeit ist nach unserem Dafürhalten jedoch weitaus besser als von ihr eingeschätzt.

In Bezug auf das Erleben der Arbeitslosigkeit sowie die Arbeitsteilung im Haushalt legen unsere Interpretationsergebnisse nahe, dass eine traditionell-konservative Fortführung der Geschlechterverhältnisse bei den Bisultanovs zu vermuten ist. Zwar sind beide gleichermaßen zum Zeitpunkt der Interviews auf Arbeitssuche, allerdings können die Ausführungen von Frau Bisultanov dahingehend gedeutet werden, dass die Arbeitslosigkeit bzw. Untätigkeit eines Mannes problematischer sei als im Falle der Frau. Die geschlechtsspezifische Aufteilung der Hausarbeit sieht Frau Bisultanov somit nicht als einen Aufgabenbereich an, der sich aufgrund der aktuellen Alltagssituation wenigstens zeitweise umverteilen ließe. Sowohl die Rollen der Geschlechter in der Reproduktionsarbeit als auch jene im (Nicht-)Erwerbsleben werden somit geschlechtsspezifisch gedeutet und entsprechend gelebt.

Die Familie kam 2007 nach Österreich und verbrachte die ersten Tage in Traiskirchen. Anschließend waren sie während des Asylverfahrens in einem Gasthof untergebracht, nicht unweit der Kleinstadt, in der auch unsere Treffen stattfanden. Nach vier Jahren erhielt die Familie subsidiären Schutz, dessen Überprüfung zum Zeitpunkt der Interviews bevorstand. Herr Bisultanov zeigt mir den Bescheid und sagt, dass sie nun unbedingt Arbeit bräuchten, denn auf der Bezirkshauptmannschaft habe man ihnen erklärt, dass sie ausgewiesen würden, wenn nicht zumindest ein Familienmitglied binnen zwölf Monaten ein halbes Jahr arbeite. Die Interpretation der Interviews legt nahe, dass diese Information die Familie in Angst versetzte und die bereits verzweifelte Arbeitssuche dramatisiert. Auf die Frage hin, ob sie eine entsprechende NGO aufgesucht hätten, um sich eine zweite juristische Mei- 
nung einzuholen, sagen sie, dass diese zu weit weg seien - in Wien oder in Graz. Die geltende Rechtslage korrespondiert nicht mit dem Informationsstand der Familie: Der subsidiäre Schutz ist ausschließlich von der Gefährdungseinschätzung im Herkunftsland abhängig. Hier zeigt sich deutlich der Einfluss, den eine einseitige Informationslage $^{9}$ in einem peripheren Gebiet für Flüchtlinge haben kann.

Auf die Bitte hin, ihre Lebensgeschichte zu erzählen, führt Herr Bisultanov unter anderem aus, dass er nach der Gewährung des subsidiären Schutzes zunächst in Wien nach einer Wohnung gesucht habe. Eine Tante wohnt im zehnten Wiener Gemeindebezirk, weshalb der Familie diese Gegend als gute Möglichkeit für einen Umzug nach Auslaufen der Grundversorgung erschien. Die Wohnungssuche führte Herr Bisultanov online durch, wobei sich rasch herausstellte, dass für Wohnungen in Wien Maklergebühren, Ablöse und Kaution anfallen. Während es für anerkannte Flüchtlinge zwar Unterstützung bei der Miete gebe, würden zusätzlich anfallende Kosten bei der Wohnungssuche nicht von staatlichen Stellen getragen, meint Herr Bisultanov. Deshalb habe er sich entschlossen, seine Suchanfrage im Netz auf das südliche Burgenland umzuändern, und sei so auf die Wohnung gestoßen - ohne derartige Zusatzkosten. Die Wohnung ist für die Familie relativ eng, hinzu kommt ein gesundheitliches Problem, da die Wohnung von Schimmel befallen ist. Das Dach scheint nicht gut gedichtet zu sein, sodass Nässe in das sichtlich sanierungsbedürftige Haus eindringt. Frau Bisultanov erzählt, dass der Hausbesitzer nicht bereit sei, notwendige Reparaturen durchzuführen. Auch die anderen Hausparteien - allesamt Zugewanderte - haben laut ihrer Schilderung dasselbe Problem.

Bei meinem zweiten Besuch kommt Herr Bisultanov auf Vorkommnisse während des Asylverfahrens zu sprechen, die ihr Alltagsleben nachhaltig beeinträchtigt haben: Während seiner Einvernahmen sei ein Übersetzer anwesend gewesen, der - laut Herrn Bisultanov nachweislich - mit dem russischen Geheimdienst zusammengearbeitet und Mitschriften der Interviews weitergegeben habe. Um seine Integrationsfortschritte in Österreich zu dokumentieren, habe Herr Bisultanov in einer solchen Einvernahme Angaben zu einem Arbeitsplatz gemacht, der ihm in Wien in Aussicht gestellt worden war. An dieser Stelle des Gesprächs unterbricht uns sein Sohn und erzählt mir, dass sein Vater in Russland per Haftbefehl gesucht werde. Am PC ruft er eine Seite mit kyrillischen Schriftzeichen auf, um mir diesen Haftbefehl zu zeigen. Sein Vater wird laut und meint, dass darin auch die Straftat erwähnt würde, die er an einem bestimmten Tag in Russland begangen haben soll - ein Datum, an dem Herr Bisultanov nachweislich in Österreich gewesen ist. Aufgrund dieser Vorkommnisse fürchte Herr Bisultanov um sein Leben. Die schon in Aussicht gestellte Arbeitsstelle habe er aus Angst vor einem Anschlag auf sein Leben nicht angetreten. Auch in der Wohnung fühle sich die Familie nicht sicher. Unsere Deutung dieser Erzählungen ist, dass die Familie ihre eigene Sicherheit unter Umständen am ländlichen Wohnort als größer einschätzt als etwa in Wien. Nur für Besuche der Tante fahren die Bisultanovs manchmal nach Wien bzw. Vater und Sohn auch an religiösen Feiertagen, um dort das nächstgelegene Gebetshaus aufzusuchen.

\footnotetext{
9 Dabei ist es an dieser Stelle nicht Aufgabe der Interpretationsarbeit, festzustellen, welche Informationen die Familie Bisultanov von der Bezirkshauptmannschaft erhalten haben. Wichtig für die Einschätzung ihrer Situation ist ihre eigene Wahrnehmung, die ihre soziale Wirklichkeit prägt.
} 


\section{Reflexion: Von der Heterogenität der Lebenslagen}

Wie postkoloniale Arbeiten zu Recht anmerken, handelt es sich beim Asylwesen um die Fortschreibung und kontinuierliche Herstellung subalterner Positionierungen von „Anderen“. Viele betroffene MigrantInnen sind Angehörige religiöser, ethnischer oder politischer Minderheiten und häufig einem doppelten kolonialen Blick durch die Mehrheitsgesellschaften ausgesetzt: Sie sind sowohl im Herkunfts- als auch im Ankunftsland von struktureller Benachteiligung und Diskriminierung betroffen. Soziale Ungleichheit wird auf diese Weise grenzüberschreitend und innerstaatlich hergestellt und verfestigt.

Soziale Ungleichheiten sind jedoch auch im Kontext der Regionen zu sehen, in denen MigrantInnen leben. Wie die autochthone Bevölkerung des Südburgenlandes erfahren MigrantInnen im Vergleich zu anderen österreichischen Regionen Benachteiligungen im Zugang zu Ressourcen, insbesondere aufgrund des eingeschränkten Ausbildungs- und Arbeitsangebots sowie der schwachen Infrastruktur (in Bezug auf Verkehrsanbindung und Beratungsangebote). Eine intersektionale Betrachtungsweise hilft hier, die spezifischen Auswirkungen für ehemalige AsylwerberInnen offenzulegen: Die regionalen strukturellen Benachteiligungen haben massivere Auswirkungen, da sie durch gruppenspezifische Diskriminierungen verstärkt werden. Die Fallbeispiele verdeutlichen, wie die Wechselwirkungen dieser mehrfachen Ungleichheit zwischen Autochthonen und MigrantInnen in zwei unterschiedlichen Ausprägungen zum Tragen kommen können: Zum einen, wenn die Nachbarschaft in Zweifel zieht, wie sich Frau Dadrian den Luxus eines Eigenheims leisten könne, zum anderen im Falle der Familie Bisultanov, die in einem Mietzinshaus in schlechtem baulichen Zustand wohnt, in dem alle anderen Parteien ebenfalls Migrationshintergrund haben. Die diskutierten Fallstudien zeigen so vor allem zweierlei: Einerseits wird deutlich, wie diese Subalternierung systemisch eingebettet ist. Andererseits konnte herausgearbeitet werden, wie sich MigrantInnen trotz dieser Umstände Handlungsspielräume erschaffen bzw. vergrößern können.

Im Unterschied zur Familie Dadrian stellt sich die Lage der Familie Bisultanov schwierig dar. Die Familie wirkt lethargisch, zumindest die Eltern und deren zwanzigjährige Tochter. Es ist anzunehmen, dass die lange Arbeitssuche und die anhaltende Wahrnehmung der Gefährdung von Leib und Leben viel Kraft kosten. Außerdem fürchten die Eltern, ihr Aufenthaltsrecht als subsidiär Schutzberechtigte aufgrund (ihrer Deutung) der Auskunft bei der Bezirkshauptmannschaft wegen der erfolglosen Arbeitssuche zu verlieren. Während nicht nur MigrantInnen von der mangelnden Verfügbarkeit von Arbeitsplätzen in der Nähe ihres burgenländischen Wohnortes betroffen sind, führt hier das Zusammentreffen unterschiedlicher Benachteiligungsfaktoren zu einer spezifischen Lebenssituation. So ist der Umstand, dass eine Familie nicht über (mindestens) ein Auto verfügen kann, in einem Gebiet ohne entsprechende öffentliche Verkehrsmittel ein entscheidender Nachteil. Dies zeigt sich insbesondere bei der Suche nach einer Arbeitsstelle. Insofern haben die spezifischen Strukturen des peripheren ländlichen Raumes einen bedeutenden Einfluss auf die Handlungsoptionen der untersuchten Personen und schränken sie mitunter in ihrer Lebensgestaltung beträchtlich ein. 
Die Fallanalysen sind daher im Sinne einer intersektionalen Betrachtungsweise auch vor dem Hintergrund der Strukturierung dieser Regionen zu sehen: Die hohe Anzahl an Wochen- und TagespendlerInnen ist ein Spezifikum des Burgenlandes. ${ }^{10}$ An dieser Stelle wird deutlich, dass es essentiell ist, sich eingehend mit der Auswahl der Untersuchungseinheiten im Sinne eines raumsensiblen Vorgehens zu befassen (Scheibelhofer 2011). Darüber hinaus zeigt sich, dass Intersektionalität nicht nur Benachteiligung aufgrund der Kategorien Geschlecht und/oder des Herkunftskontextes umfasst, sondern auch die Beschaffenheit des regionalen Raums innerhalb eines privilegierten nationalen Kontextes bedeutsam sein kann (Oehme 2015). Diesen Besonderheiten des Regionalen als eine Dimension intersektionaler Analysen wurde bisher kaum Beachtung geschenkt. Gerade dieser Aspekt kommt im untersuchten Gebiet in noch größerem Maße für die untersuchten Flüchtlinge zum Tragen, da sie nicht wie der Großteil der autochthonen Bevölkerung imstande sind, die Mobilität über einen PKW zu finanzieren. Mit dieser Form der Mobilität sind jedoch gerade in peripheren Gebieten wichtige Chancen der Teilhabe verbunden, wie etwa das Erreichen eines Arbeitsplatzes. Intersektionalität bedeutet damit auch in diesem Falle nicht die Analyse eines additiven Nebeneinanders der Benachteiligungen aufgrund mehrerer sozialer Kategorien, sondern ein sich gegenseitig verstärkendes, interdependentes Ineinanderwirken verschränkter Kategorien (Hancock 2007). Dieses komplexe Zusammenspiel ist dabei nicht nur auf Makro- und Mesoebene gesellschaftlicher Strukturen zu beobachten, sondern auch auf der Ebene von Individuen, Familien und/oder Haushalten. Zwischen diesen Ebenen ist eine wechselseitige Beeinflussung konzeptionell anzunehmen, die soziale Ungleichheiten noch weiter verstärkt (vgl. u. a. Winker and Degele 2011).

Die intersektionale Herangehensweise hilft auch dabei, einen Konnex zwischen der eingeschränkten Infrastruktur an Beratungsangeboten für Flüchtlinge in derart peripheren Regionen (wenngleich hierbei auf den punktuellen Ausbau aufgrund des Engagements einiger NGOs verwiesen sei) und sozialer Ungleichheit herzustellen. Im Gegensatz zu den mitunter weitreichenden Konsequenzen - für Familie Bisultanov ergibt sich durch die geschilderte Situation eine spezifische Verletzungsdisposition - sind soziale Praxis und das damit verbundene Wissen um qualitativ hochwertige Beratung für MigrantInnen in Städten auch geografisch eher zugänglich (UNHCR 2013).

Im Gegensatz dazu steht Familie Dadrian, die sich zwischen kommunikativer Anpassungsfähigkeit und kreativer Widerständigkeit ihren Weg spurt. Das Eigenheim und dessen Einrichtung fungiert für die Familie als Statussymbol, wobei sie dem Wertesystem der autochthonen Bevölkerung weitgehend folgt. Diese bringt den Zugewanderten großteils Misstrauen entgegen und bezweifelt, dass sich Flüchtlinge ohne die Anwendung illegitimer Mittel ein Eigenheim leisten können. Erfahrungen mit der direkten Nachbarschaft können auch im Zusammenhang mit breiteren Diskursen gelesen werden: So wird Flüchtlingen üblicherweise entweder ein Opferstatus als passive HilfeempfängerInnen zugewiesen, oder sie werden unter Generalverdacht gestellt, Sozialleistungen missbräuchlich zu empfangen (Langthaler

${ }_{10}$ Der Anteil der BerufspendlerInnen im Burgenland verzeichnet eine steigende Tendenz. Pendelten 1971 nur rund $43 \%$ der Erwerbstätigen, so waren es 2011 bereits $74 \%$ (Statistik Austria 2013). 
und Sohler 2008, S. 17). Frau Dadrian schildert im Interview auch institutionelle Hürden in Bezug auf ihr Leben im peripheren Raum, etwa um den Führerschein zu erlangen. Das Verhältnis zu VertreterInnen relevanter Institutionen stellt sich bei Frau Dadrian nicht zuletzt aufgrund ihrer außergewöhnlichen sozialen und kommunikativen Kompetenzen als verhandelbar dar: Sie ist erfolgreich darin, mittels eines aktiv gepflegten Netzwerkes strukturellen Benachteiligungen zu entkommen.

Aufgrund der hier vorgestellten ersten Ergebnisse aus der Pilotphase unseres Forschungsprojektes ist anzunehmen, dass Flüchtlinge in der Peripherie spezifische Handlungspraktiken entwickeln, um die organisierte Desintegration (Täubig 2009) der Lebensphase des Asylverfahrens hinter sich zu lassen. Einige gesellschaftliche Teilbereiche scheinen dabei leichter zugänglich, wie etwa die Mitgliedschaft in einer Musikkapelle oder auch das Aufrechterhalten hilfreicher Freundschaften zur autochthonen Bevölkerung. Am Arbeitsmarkt aber scheinen diese Handlungsräume für die Gestaltung der Lebensbedingungen durch strukturelle Benachteiligungen und nicht zuletzt durch die Infrastruktur im ländlichen Bereich begrenzt. Diese ersten Fallbeispiele eines größeren Forschungszusammenhangs deuten somit an, wie soziale und vor allem kommunikative Ressourcen auch innerhalb einer Familie ungleich verteilt sein können. Die Generationen- und Geschlechterverhältnisse zeigen sich als komplex und benötigen dringend einer weiteren Untersuchung. So wird in beiden Familien von traditionellen Geschlechterrollen ausgegangen, die jedoch im Falle der Familie Dadrian moduliert werden, wenn Herr Dadrian aufgrund der Berufstätigkeit seiner Frau Hilfstätigkeiten im Haushalt übernimmt, die weiblich konnotiert sind. Es wird Aufgabe eines künftigen Projektverlaufs sein, zu beleuchten, wie Erfahrungen aus dem Migrationsverlauf, des Asylverfahrens und der Etablierung eines Alltagslebens in einer peripheren Region ineinandergreifen.

Open access funding provided by University of Applied Sciences Burgenland.

Open Access Dieser Artikel wird unter der Creative Commons Namensnennung 4.0 International Lizenz (http://creativecommons.org/licenses/by/4.0/deed.de) veröffentlicht, welche die Nutzung, Vervielfältigung, Bearbeitung, Verbreitung und Wiedergabe in jeglichem Medium und Format erlaubt, sofern Sie den/die ursprünglichen Autor(en) und die Quelle ordnungsgemäß nennen, einen Link zur Creative Commons Lizenz beifügen und angeben, ob Änderungen vorgenommen wurden.

\section{Literatur}

Ammer, Margit. 2011. Access to Wage-Earning Emploment by Third-Country Nationals in Need of Protection in Austria: Conformity with Human and Fundamental Rights Standards? Dissertation. Wien.

Amt der burgenländischen Landesregierung. 2013. Sozialbericht 2011/2012. Burgenland: Abteilung 6. http://www.burgenland.at/fileadmin/user_upload/Downloads/Gesundheit_und_Soziales/Soziales/ Berichte_Publikationen/Sozialberichte/2932_Burgenlaendischer_Sozialbericht_2011-2012.pdf. Zugegriffen: 28. April 2016.

Brunner, Karl-Michael, Gerhard Jost, und Manfred Lueger. 1994. Flüchtlingsunterbringung in einer Kleingemeinde: eine Fallstudie zur sozialen Dynamik von Migrationsprozessen. Soziale Welt 45:125-146.

Bundesministerium für Inneres. 2014. Asylstatistik 2013. Wien: Sektion III Recht.

Burtscher, Simon. 2012. Integrationsdiskurse und Integrationspolitik in Vorarlberg - eine prozesssoziologische Perspektive. In Migration und Integration - wissenschaftliche Perspektiven aus Österreich Jahrbuch 1/2011, Hrsg. Julia Dahlvik, Heinz Fassmann, und Wiebke Sievers, 283-302. Göttingen, Wien: V \& Unipress, Vienna University Press. 
Chakrabarty, Dipesh. 2000. Provincializing Europe: Postcolonial Thought and Historical Difference. Princeton: Princeton University Press.

Charmaz, Kathy. 2006. Constructing Grounded Theory. A Practical Guide Through Qualitative Analysis. Los Angeles, London: SAGE.

Conway, J. (2011). Cosmopolitan or colonial? The world social forum as 'contact zone'. Third World Quarterly 32(2):217-236.

European Commission. 2014. Common European Asylum System (CEAS). http://ec.europa.eu/dgs/homeaffairs/what-we-do/policies/asylum/index_en.htm. Zugegriffen: 28. April 2016.

European Parliament, und Council of the European Union. 2011. Directive 2011/95/EU of the European Parliament and of the Council of 13 December 2011 on standards for the qualification of thirdcountry nationals or stateless persons as beneficiaries of international protection, for a uniform status for refugees or for persons eligible for subsidiary protection, and for the content of the protection granted (recast)

Fritsche, Andrea. 2012. Zeit. Macht. Flüchtlinge. Und Flüchtlinge machen Zeit. Konzeptionen biographischer Zeiten im Asylkontext. SWS Rundschau 4:362-388.

Ha, Noa. 2014. Perspektiven urbaner Dekolonisierung: Die europäische Stadt als ,Contact Zone‘. sub/ urban 2(1):27-48.

Hancock, Ange-Marie. 2007. When Multiplication Doesn't Equal Quick Addition: Examining Intersectionality as a Research Paradigm. Perspectives on Politics 5(1):63-79.

Hermanns, Harry. 1992. Die Auswertung narrativer Interviews. Ein Beispiel für qualitative Verfahren. In Analyse verbaler Daten. Über den Umgang mit qualitativen Daten, Hrsg. Jürgen Hoffmeyer-Zlotnik, 111-142. Opladen: Westdeutscher Verlag.

Jenkins, Richard. 1997. Rethinking Ethnicity. Arguments and Explorations. London: SAGE.

Kirpitchenko, Liudmila, und Lejla Voloder. 2014. Insider Research on Migration and Mobility: International Perspectives on Researcher Positioning. Burlington, Vermont: Ashgate.

Kraler, Albert, Christina Hollomey, Christoph Hurich, Andrea König, und Gerhard Muzak. 2013. Family Reunification: a barrier or facilitator of integration? Country Report Austria. Vienna: European Commission.

Langthaler, Herbert, und Karin Sohler. 2008. Politische Partizipation und Repräsentanz von Flüchtlingen und AsylwerberInnen in der EU: Synthese der Fallstudien in Österreich, Frankreich und der Tschechischen Republik. Wien: BMWF. Project >node.

Luimpöck, Sabrina. 2015. Exklusion von Asylsuchenden als Menschenrechtsverletzung. Sozialarbeit in Österreich 3:18-20.

Nayar, Pramod K. 2010. Postcolonialism. A Guide for the Perplexed Series. New York: Continuum.

Neuwirth, Markus. 2007. Integration von Flüchtlingen in Niederösterreich - Wege für die Zukunft. München: GRIN.

Oehme, Ulrike. 2015. Diversity im ländlichen Raum. Eine ethnographische Untersuchung zur Konstruktion von Unterschieden in der Regionalentwicklung. Dissertation. Hildesheim: Universität Hildesheim.

Pantuček, Peter. 2012. Soziale Diagnostik. Verfahren für die Praxis Sozialer Arbeit, 3. Aufl., Wien, Köln, Weimar: Böhlau.

Reichel, David, und Alina Cibea. 2014. Die Verwendung von Integrationsindikatoren zur Messung der Wirkung von Integrationspolitik in Europa. In Die Verwendung von Integrationsindikatoren zur Messung der Wirkung von Integrationspolitik in Europa, Hrsg. Julia Dahlvik, Christoph Reinprecht, und Wiebke Sievers, 87-104. Göttingen, Wien: V\&R Unipress - Vienna University Press.

Scheibelhofer, Elisabeth. 2011. Raumsensible Migrationsforschung: methodologische Überlegungen und ihre empirische Relevanz für die Migrationssoziologie. Wiesbaden: Springer.

Scheibelhofer, Elisabeth. 2016. Reflecting on Spatiality in European Migration Research: From Methodological Nationalism to Space-Sensitive Observations of Social Transformations. In An Anthology of Migration and Social Transformation, Hrsg. Anna Amelina et al., 73-86. Berlin: Springer.

Servent, Ripoll, Ariadna Trauner, und Florian Trauner. 2014. What Kind of Impact is Austria Exposed to? Analysing EU Asylum Law. In Migration und Integration - wissenschaftliche Perspektiven aus Österreich, Hrsg. Julia Dahlvik, Christoph Reinprecht, und Wiebke Sievers, 319-333. Göttingen, Wien: V\&R Unipress - Vienna University Press.

Sonn, Christopher C., und Amy F. Quayle. 2013. Developing Praxis: Mobilising Critical Race Theory in Community Cultural Development. Journal of Community \& Applied Social Psychology 23:435-448.

Statistik Austria. 2013. Census 2011 Burgenland. Ergebnisse zur Bevölkerung aus der Registerzählung. Wien: Statistik Austria.

Täubig, Vicki. 2009. Totale Institution Asyl. Empirische Befunde zu alltäglichen Lebensführungen in der organisierten Desintegration. Weinheim: Juventus. 
UNHCR. 2013. Facilitators and Barriers. Refugee Integration in Austria. European Refugee Fund of the European Commission. In Refugee Integration. Capacity and Evaluation in Europe, Hrsg. UNHCR. Vienna: UNHCR.

UNHCR. 2014. 2014 UNHCR regional operations profile - Northern, Western, Central and Southern Europe. http://www.unhcr.org/pages/49e48e5f6.html. Zugegriffen: 28. April 2016.

Winker, Gabriele, und Nina Degele. 2011. Intersectionality as multi-level analysis: Dealing with social inequality. European Journal of Women's Studies 18(1):51-66.

Elisabeth Scheibelhofer Assoz.-Prof.in PD Dr.in, arbeitet als assoziierte Professorin am Institut für Soziologie der Universität Wien. Ihre Forschungsarbeiten fokussieren auf die Themen qualitative Methodologien und Methoden sowie Migration, transnationale Mobilität und Flucht. Sie arbeitet momentan an dem internationalen Forschungsprojekt TRANSWEL (2015-2018), das sich mit der Portabilität sozialer Rechte von EU-MigrantInnen beschäftigt. Zu ihren Publikationen zählen internationale Beiträge zu qualitativen Methoden sowie Bücher und Zeitschriftenartikeln, die sich mit Fragen der Mobilität und Migration im Kontext der Zweiten Moderne auseinandersetzen.

Sabrina Luimpöck Mag.a (FH) BA, arbeitet im Rahmen ihrer Dissertation am Institut für Soziologie (Universität Wien) zu Erwerbsbiografien tschetschenischer Flüchtlinge und geht der Frage nach, wie Exklusion Netzwerke und Identitätskonstruktionen beeinflusst. Nach ihren Studien der Sozialen Arbeit und Slawistik lehrt und forscht sie derzeit im Burgenland und in Wien. Ihre Schwerpunkte sind Handlungsräume und -grenzen Sozialer Arbeit im Asylbereich sowie das Zusammenspiel familiärer, nachbarschaftlicher und institutioneller Netzwerkressourcen in den Bereichen Vereinbarkeit von Beruf und Familie sowie Altern. 